\title{
Practice gaps and barriers to optimal care of hematologic malignancies in the United States
}

Suzanne Murray, BA, ${ }^{a}$ Kevin L Obholz, PhD,b Andrew D Bowser, ELS, CCMEP,b Jim Mortimer, BA, ${ }^{\mathrm{b}}$ Patrice Lazure, MSc, ${ }^{a}$ Eric Peterson, EdM, FACEHP, ${ }^{\mathrm{c}, \mathrm{d}}$ James O Armitage, $\mathrm{MD},{ }^{\mathrm{e}}$ and B Douglas Smith, $\mathrm{MD}^{\mathrm{f}}$

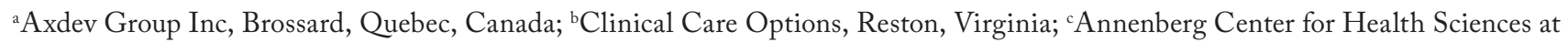
Eisenhower, Rancho Mirage, California; ${ }^{\mathrm{d} A}$ merican Academy of Physician Assistants, Alexandria, Virginia; ${ }^{\mathrm{c}}$ The Nebraska Medical Center in Omaha, Nebraska; and ${ }^{\mathrm{f} K i m m e l ~ C a n c e r ~ C e n t e r ~ a t ~ J o h n s ~ H o p k i n s, ~ B a l t i m o r e, ~ M a r y l a n d ~}$

Background Treating patients with hematologic malignancies can be challenging for physicians because of the rapidly evolving standards of care and relatively low incidence of these diseases.

Objective To identify clinical challenges among hematologists and medical oncologists regarding the provision of care to patients with chronic myeloid leukemia (CML), acute lymphoblastic leukemia (ALL), or B-cell lymphomas.

Methods Hematologists and medical oncologists in active practice in the United States and who have a case load of $\geq 1$ patient a year with CML, ALL, or B-cell lymphoma were recruited. The initial qualitative phase consisted of an online case-based survey followed by an interview exploring the contextual and behavioral factors that influence treatment decisions $(n=27)$. The analysis of qualitative data then informed a quantitative phase, in which 121 participants completed an online survey composed of case vignettes, multiple choice, and semantic differential rating scale questions. The respondents' answers were compared with recommendations from treatment guidelines and faculty experts.

Results A higher frequency of bone marrow biopsies was reported compared with expert faculty recommendations by $74 \%$ of oncologists. Many respondents failed to recognize the clinical relevance of BCR-ABL mutations other than T315I. Respondents reported perceiving difficulties in individualizing treatment and interpreting response to treatment in patients with ALL and B-cell lymphomas. Fewer than $30 \%$ of respondents recognized the mechanisms of action of 5 of the 9 promising investigational agents presented.

Limitations Participant self-selection bias is a possibility because participation was voluntary. Practice gaps are not based on clinical data, but hypothetical case situations and self-report.

Conclusions Findings from this study can guide education to address the identified challenges in caring for patients with hematologic malignancies and improving patient care.

Funding This needs assessment was financially supported with an educational research grant from Pfizer Medical Education Group to the Annenberg Center for Health Sciences at Eisenhower.

$\mathrm{T}$ The care of patients with chronic myeloid leukemia (CML), acute lymphoblastic leukemia (ALL), and B-cell lymphomas present clinical challenges for many clinicians in the United States. ${ }^{1}$ Many new agents and therapeutic strategies are under clinical investigation or have been recently approved for use against these hematologic malignancies and treatment selection is shifting from a one-size fits all approach to an individualized approach based on patient and tumor characteristics. ${ }^{2-5}$ Community-based clinicians often have limited experience with low prevalence diseases and need ongoing education and training to understand rapidly evolving standards of care. ${ }^{6}$
System reforms are also adding pressure to the clinical decisions of hematologists and medical oncologists. The US Patient Protection and Affordable Care Act (PPACA) includes a provision stating that Medicare reimbursements will move from fee-for-service to bundled payments, whereby a single payment is paid for a predefined episode of care, rather than a series of payments based on each specific service provided. ${ }^{7}$ In that context, physicians are incentivized to reach greater efficiency and improve their clinical performance, which could be achieved with a better understanding of their own challenges in treatment decisions.

The goal of this national practice assessment was

Accepted for publication March 27, 2014. Correspondence: Suzanne Murray; murrays@axdevgroup.com. Disclosures: Dr Armitage has received consulting fees from Ziopharm, Seattle Genetics, Spectrum, GlaxoSmithKline, Genentech, and Roche, and is on the board of directors of Tesaro. Dr Smith has received consulting fees from Ariad, Bristol-Myers Squibb, and Novartis. The other authors have no potential conflicts of interest to disclose. JCSO 2014;12:329-338. (2014 Frontline Medical Communications. DOI 10.12788/jcso.0073 
to better understand current clinical challenges and the potential barriers to optimal care experienced by US hematologists and medical oncologists who treat patients with CML, ALL, or B-cell lymphomas. Findings from this assessment will help identify areas in which these specialists need to reflect on their own practice and will help better inform the design and deployment of future continuing medical education activities and performance improvement interventions.

\section{Methods}

This assessment integrated the collection and analysis of qualitative and quantitative data deployed in 2 consecutives phases, in which an initial qualitative exploratory phase (March-May 2013) informed a subsequent quantitative confirmatory phase (May-June 2013) in a mixed-methods framework. ${ }^{8}$ The approach draws on the strengths of each phase: the depth of qualitative data and the analytic power of quantitative data collection. ${ }^{8}$ Source triangulation was used to increase the validity and trustworthiness of findings. ${ }^{9}$ Triangulation consisted of combining different research methodologies (qualitative, quantitative) and different data collection methods (interviews, surveys). Two distinct independent ethical approvals (IRB Services, Boca Raton, FL for qualitative phase and Eisenhower Medical Center Institutional Review Board for quantitative phase) were obtained to ensure informed consent, protection, and confidentiality of participants, as per national guidelines and policies. ${ }^{10}$

\section{Research tool design}

A literature review and internal data from coauthors were used to generate hypotheses about gaps in knowledge, skills, and clinical confidence among US hematologists and medical oncologists. Hypotheses and consultation with 2 nationally recognized experts in hematologic malignancies informed the design of a 15-minute casebased online survey and a 45-minute, semistructured, interview guide. The interviews focused on the challenges experienced by providers as they answered the case-based questions, and on the contextual and behavioral factors that influence their clinical reasoning process. Findings from the qualitative phase and further consultation with experts informed the design of a 15-20 minute, online, quantitative survey deployed in phase 2 of the study. The survey consisted of case vignettes, multiple choice questions, and semantic differential rating scale questions (online file 1).

\section{Recruitment and data collection}

Invitations to participate in both phases of the study were sent through email to a list of 11,696 hematologists and medical oncologists who were members of Clinical Care
Options. Invitations included a web link at which interested participants could learn about the study, sign a consent form, and answer eligibility questions before being redirected to complete the phase 1 or phase 2 survey.

A combination of criterion sampling and maximum variation sampling ${ }^{11}$ was used to include a sample with a mix of years of practice and practice settings, ensuring a broad spectrum of perspectives on the reality of care. Eligible participants for the qualitative phase of the study had to be actively practicing in oncology in the United States, have a case load of at least 2 patients a year with CML, ALL, or B-cell lymphomas, and a minimum of 10 patients a year for all 3 conditions combined. In the quantitative phase, the case load inclusion criteria was reduced from 2 per condition to a combined total of at least 1 case a year to allow for identification of challenges in the group of practitioners most likely to be unfamiliar with these relatively rare diseases.

\section{Analysis plan}

A subset of transcribed interviews was coded and analyzed using NVivo qualitative data analysis software (QSR International Pty Ltd, Version 7, 2006). The qualitative analysis approach draws from the principles of both thematic analysis ${ }^{12}$ and directed content analysis. ${ }^{13}$ More specifically, the approach included 4 steps: identification of predetermined codes, based on literature; coding of data based on step 1; analysis of data that could not be coded and refinement of coding tree; and identification of emerging themes with substantial data.

The data collected from the online cases in phase 1 and from the quantitative survey in phase 2 were analyzed using SPSS 12.0 software (SPSS, Chicago, IL). Answers were compared with optimal or acceptable answers (as identified by treatment guidelines ${ }^{14-17}$ and experts); differences between optimal and actual practice were considered to be a practice gap. ${ }^{18}$ Triangulation of data was performed to link potential causalities reported in the interviews, to the practice performance gaps identified from the quantitative phase. Subgroup differences (by years of practice, practice types, or case load) were calculated using Pearson's chi-square test (hereafter referred to as chi-square).

\section{Results}

\section{Sample size and demographics}

For the qualitative phase of the study, 27 eligible physicians completed the case-based survey and were subsequently interviewed. For the quantitative phase of the study, 121 eligible respondents were included in the analysis. Sample demographics are presented in Table 1. Respondents were evenly distributed for years of practice and represented a variety of practice settings, including academic medical 
centers (38\%) and group or solo practices (34\%). All of the respondents had at least $1 \%$ of their case load represented by the 3 malignancies combined, and more than half of the sample (53\%) reported that patients with CML, ALL, or B-cell lymphomas represented more than $20 \%$ of their case load. The samples of respondents from the qualitative and quantitative phases were not statistically significantly different from each other.

\section{Identified practice performance gaps}

A summary of the gaps identified is included in online file 2. Gaps most indicative of competencies needed for hematologists and medical oncologists to individualize treatment according to patient and tumor characteristics are detailed here. Qualitative quotes illustrating these gaps are included in online file 3 .

\section{First-line treatment of chronic-phase CML}

There was a discrepancy in the choice of first-line tyrosine kinase inhibitor (TKI) therapy for chronic phase CML between the respondents' answers and those suggested by the expert faculty. Most of the respondents $(62 \%)$ indicated that imatinib is their preferred first-line therapy in this situation, whereas the faculty recommended dasatinib or nilotinib (Table 2, Question 1).

Respondents from academic settings were significantly more likely to select nilotinib than were their colleagues from nonacademic settings (19\% vs 10\%, respectively; chisquare; $P=.039$ ). The most frequently reported reason for the use of imatinib as first-line therapy for chronic phase CML was that imatinib is still perceived as the standard of care and is the agent with which they have the most clinical experience.

When asked their level of agreement with the statement Early molecular responses to TKI therapy correlate with long-term clinical outcomes for patients with chronic phase CML, 33\% of respondents selected the same level of agreement as the expert faculty (6 on a scale from 1 = strongly disagree to 7 = strongly agree), and $18 \%$ of respondents were in disagreement or neutral with the statement. For the statement Achieving a major molecular response (MMR) to TKI therapy substantially decreases the patient's risk of disease progression, $38 \%$ of respondents selected the same level of agreement as the expert faculty ( 7 on the same scale), and $13 \%$ of respondents were in disagreement or neutral with that statement.

\section{Monitoring response to first-line TKI therapy}

When asked to describe their timing and frequency of cytogenetics by bone marrow biopsy to assess patient response to first-line TKI therapy, a substantial proportion of respondents (74\%) selected a higher frequency of bone marrow biopsies than recommended by the expert faculty (Table 2, Question 2). When asked to describe the timing and frequency of peripheral blood assessment of BCRABL transcripts by quantitative polymerase chain reaction (QPCR), 40\% matched the expert response (Table 2, Question 3). A significant difference in response was observed by practice setting wherein a higher proportion of respondents from academic centers (52\%) were aligned with the expert faculty's response, compared with those from nonacademic settings (31\%; chi-square; $P=.030$ ).

Respondents' ability to interpret molecular and cytogenetic analyses to inform treatment decisions was assessed through the use of 8 different scenarios (Table 3). For 7 of the 8 assessments, level of respondent agreement with the study experts and guideline recommendations was less than 50\%. For a patient whose monitoring 


\section{Original Report}

TABLE 2 Description of selected cases and answers to selected clinical and case-based questions ${ }^{a}$

1. Which of the following TKI do you most often recommend as first-line therapy to treat newly diagnosed CP CML? ( $n=121)$
A. Bosutinib
B. Dasatinib
C. Imatinib
D. Nilotinib
E. Ponatinib

n (\%)

0

$29(24.0)$

$81(61.8)$

$21(17.4)$

0

\section{Timing of bone marrow cytogenetic analysis for patients with CML after assessment at diagnosis?} ( $n=121$ )

A. Every 3 mo after initiating therapy, regardless of response

B. Every 6 mo after initiating therapy, regardless of response

C. Every 12 mo after initiating therapy, regardless of response

D. 3 mo after initiating therapy and again at $12 \mathrm{mo}$ if no CCyR or MMR at Month 3

E. Only after evidence of disease progression and/or TKI failure

3. Timing for assessing BCR-ABL transcript levels by QPCR using the IS for your patients with CML after assessment at diagnosis? $(n=121)$

A. Every 3 mo after initiating therapy, regardless of response

B. Every 3 mo after achieving CCyR

C. Twice yearly, regardless of response

D. Every 3 mo after initiating therapy and then discontinue after MMR is achieved

E. Other

$4(3.3)$

\section{How would you treat this patient in your current practice? $(n=121)$}

\section{CASE A}

- Patient with CP CML

- Has taken $400 \mathrm{mg}$ imatinib for $24 \mathrm{mo}$

- At 24 mo, BCR-ABL QPCR is positive at $2.23 \%$ using the IS

- Bone marrow analysis are consistent with chronic-phase disease

- Cytogenetics show $\$(9 ; 22)(q 34 ; q 11.2)$ in 3 out of 20 cells

- A T359I mutation (not T315I) is identified in $A B L$

A. Allogeneic bone marrow transplantation
B. Continue imatinib at current dose
C. Consider a clinical trial
D. Increase imatinib dose to $800 \mathrm{mg}$
E. Switch therapy to bosutinib
F. Switch therapy to dasatinib
G. Switch therapy to nilotinib
H. Switch therapy to omacetaxine
I. Switch therapy to ponatinib

5. What would you recommend for this patient if a T315I (instead of T359l) was identified in ABL? ( $\mathrm{n}=121)$

\begin{tabular}{|c|c|}
\hline $\mathbf{n}(\%)$ & $\mathbf{n}(\%)$ \\
\hline $7(5.8)$ & $12(9.9)$ \\
\hline $7(5.8)$ & $2(1.7)$ \\
\hline $13(10.7)$ & $15(12.4)$ \\
\hline $17(14.0)$ & $3(2.5)$ \\
\hline $13(10.7)$ & $13(10.7)$ \\
\hline $41(33.9)$ & $4(3.3)$ \\
\hline $22(18.2)$ & $8(6.6)$ \\
\hline $1(0.8)$ & $6(5.0)$ \\
\hline $15(12.4)$ & $69(57.0)$ \\
\multicolumn{3}{c}{ Continued on next page } \\
\hline
\end{tabular}

results were $\mathrm{BCR}-\mathrm{ABL} / \mathrm{ABL}=15 \%$ in the International Scale and 17/20 (Ph)-positive metaphases at 3 months, the expert faculty recommended that Sometimes a switch in therapy would be necessary in this scenario, whereas $40 \%$ of respondents indicated that they would rarely do so (Table 3, clinical scenario A). Less experienced hematologists and medical oncologists were more likely to respond Rarely to that question compared with the more experienced physicians ( $56 \%$ vs $28 \%$, respectively), whereas a higher proportion of those with more than 10 
TABLE 2 continued

\section{CASE B}

- 55-year-old man with abdominal pain, vomiting, and a 30-pound weight loss

- Was found to have a palpable large abdominal mass:

- Biopsy showed diffuse large B-cell lymphoma

- PET-CT scan showed a 20-cm mass, retroperitoneal nodes, and a right iliac node with an SUV maximum of 20

- Bone marrow was negative;

- Lactate dehydrogenase was twice the maximum normal;

- Patient promptly received 1 cycle of CHOP-R with resolution of symptoms and marked reduction in size of mass;

- Ki-67 of $90 \%$ and FISH studies showed a MYC rearrangement after drugs administration.

\section{CASE C}

- 70-year-old man;

- Just diagnosed with mantle cell lymphoma;

- Has involved nodes above and below the diaphragm and $10 \%$ involvement of the bone marrow.
6. What would you recommend if gene rearrangement studies became available for this patient and showed an activated B-cell lymphoma genotype? $(n=120)^{b}$

n (\%)

A. Add bortezomib to either CHOP-R or EPOCH-R

35 (29.2)

B. Change to R-DHAP or RICE

$22(18.3)$

C. Add lenalidomide to CHOP-R

$12(10.0)$

D. Plan to proceed with an autologous transplantation after

$36(30.0)$

E. Other

$15(12.5)$

7. How would you treat this patient if the FISH results showed both a MYC and a BCL2 rearrangement? $(n=120)^{b}$

A. Complete CHOP-R and consider an autologous BMT in first complete 45 (37.5) remission

B. Change to ACVBP-R

C. Add bortezomib to CHOP-R

D. Change to EPOCH-R and consider an autologous BMT in first complete remission

48 (40.0)

E. Other

$7(5.8)$

\section{How would you treat this patient in your current practice?}

(n = 121)

A. CVP-R

$3(2.5)$

B. CHOP-R

$54(44.6)$

C. CHOP-R-bortezomib

D. Modified hyperCVAD

$15(12.4)$

E. Bendamustine and rituximab

F. Chlorambucil-R

G. Other

ACVBP-R, doxorubicin, cyclophosphamide, vindesine, bleomycin, prednisone, plus rituximab; BMT, bone marrow transplantation: $C$ C $\mathrm{R}$, complete cytogenic response: $C H O P-R$, cyclophosphamide, hydroxydaunorubicin, oncovin, prednisone, plus rituximab; CML, chronic myeloid leukemia; CP-CML, chronic phase chronic myeloid leukemia; CVAD, cyclocyclophosphamide, hydroxydaunorubicin, oncovin, prednisone, plus rituximab; CML, chronic myeloid leukemia; CP-CML, chronic phase chronic myeloid leukemia; $C V A D$, cyclo-
phosphamide, vincristine, doxorubicin, examethoasone; CVP-R, cyclophosphamide, vincristine, prednisone, plus rituximab; EPOCH-R, etoposide, prednisone, oncovin, cyclophosphosphamide, vincristine, doxorubicin, examethoasone; CVP-R, cyclophosphamide, vincristine, prednisone, plus rituximab; EPOCH-R, etoposide, prednisone, oncovin, cyclophos-
phamide, hydroxydaunorubicin, plus rituximab; FISH, florescence in situ hybridization; MMR, mismatch repair; PET-CT, positron emission tomography-computed tomography; phamide, hydroxydaunorubicin, plus rituximab; FISH, florescence in situ hybridization; MMR, mismatch repair; PET-CT, positron emission tomography-computed tomography;
QPCR, quantitative polymerase chain reaction; R, rituximab; R-DHAP, rituximab plus dexamethasone, cytarabine, cisplatin; RICE, rituximab, ifosfamide, carboplatin, etoposide; SUV, standardized uptake value; TKI, tyrosine kinase inhibitor

aFaculty best answers are shaded green; other acceptable answers shaded blue. bOne patient did not answer this question.

years of experience compared with those with less than 10 years of experience were aligned with faculty's answer (46\% vs $24 \%$; chi-square; $P=.017$ ). For clinical scenario $\mathrm{G}$ (Loss of CCyR at any time point), $75 \%$ of respondents with at least 10 cases of CML, ALL, and B-cell lymphomas a year were aligned with faculty's response and answered Definitely, while 39\% of those with fewer than 10 cases a month agreed with the expert recommendation (chi-square; $P=.037$ ). Qualitative interviews revealed that many respondents did not understand the optimal timing of or how to read and interpret QPCR response data and therefore are challenged to determine when a change in therapy is indicated.

Therapeutic strategies to overcome TKI resistance The survey respondents were presented with 2 cases in which therapeutic strategies were needed to overcome imatinib resistance (Table 2, Questions 4 and 5). For the scenario described in Question 5, that included a mutation for which multiple agents are expected to be effective, 34\% of respondents selected the therapeutic strategy that was considered optimal for this patient by the faculty, and just over $60 \%$ selected one of the multiple treatment options recommended in treatment guidelines. Respondents from academic settings were more likely than those from nonacademic settings to have selected Switch therapy to nilotinib ( $28 \%$ vs $11 \%$, respectively; chi-square; $P=.018$ ).

For the scenario described in Question 5 (a T315I mutation), $57 \%$ of respondents selected Switch therapy to ponatinib, and $10 \%$ selected allogeneic bone marrow transplantation for this scenario. Respondents from nonacademic settings were more likely than those from academic set- 


\section{Original Report}

\begin{tabular}{|c|c|c|c|c|c|}
\hline Clinical scenario & $\mathbf{n}$ & $\begin{array}{l}\text { Definitely, } \\
\text { n \% }\end{array}$ & $\begin{array}{l}\text { Sometimes, } \\
\text { n \% }\end{array}$ & $\begin{array}{l}\text { Rarely, } \\
\text { n \% }\end{array}$ & $\begin{array}{l}\text { Unsure, } \\
\text { n \% }\end{array}$ \\
\hline BCR-ABL/ABL $=15 \%$ IS, $17 / 20 \mathrm{Ph}$-positive metaphases at $3 \mathrm{mo}$ ? & 120 & $26(21.7)$ & $43(35.8)$ & $48(40.0)$ & $3(2.5)$ \\
\hline BCR-ABL/ABL $=4 \% \mathrm{IS}, 6 / 20 \mathrm{Ph}$-positive metaphases at $12 \mathrm{mo}$ ? & 121 & $59(48.8)$ & $33(27.3)$ & $27(22.3)$ & $2(1.7)$ \\
\hline BCR-ABL/ABL of $1 \%$ IS, 0/20 Ph-positive metaphases at $12 \mathrm{mo}$ ? & 119 & $10(8.4)$ & $31(26.1)$ & $75(63.0)$ & $3(2.5)$ \\
\hline $\begin{array}{l}\text { CCyR at Month } 18 \text {, but no MMR (BCR-ABL/ABL }=0.15 \% \text { and } \\
0.22 \% \text { IS at Months } 15 \text { and } 18) \text { ? }\end{array}$ & 119 & $32(26.9)$ & 47 (39.5) & $36(30.3)$ & $4(3.4)$ \\
\hline $\begin{array}{l}\text { CCyR at Month } 18 \text {, but no MMR (BCR-ABL/ABL }=0.18 \% \text { and } \\
1.05 \% \text { IS at Months } 15 \text { and } 18) \text { ? }\end{array}$ & 120 & $45(37.5)$ & $53(44.2)$ & $18(15.0)$ & $4(3.3)$ \\
\hline CCyR by Month 12 , but no MMR by Month 24 ? & 119 & $39(32.8)$ & $60(50.4)$ & $18(15.1)$ & $2(1.7)$ \\
\hline Loss of CCyR at any time point? & 120 & $84(70.0)$ & 27 (22.5) & $7(5.8)$ & $2(1.7)$ \\
\hline Loss of MMR at any time point? & 120 & $48(40.0)$ & $60(50.0)$ & $7(5.8)$ & $5(4.2)$ \\
\hline
\end{tabular}

tings to have selected Switch therapy to bosutinib (17\% vs $2 \%$, respectively; chi-square; $P=.014$ ); but more respondents from academic settings selected Switch therapy to ponatinib ( $76 \%$ vs $44 \%$; chi-square; $P=.001$ ).

In phase I, more than $50 \%$ of respondents selected ponatinib for the patient scenario described in Question 5, when the T359I mutation was not specifically mentioned as not being the more clinically relevant T315I. With the clarification added in the phase 2 survey, $12.4 \%$ selected ponatinib. Data from qualitative interviews indicated a lack of knowledge and understanding of how to interpret mutation reports in CML.

\section{Individualizing first-line therapy for patients with B-cell lymphomas}

The assays reported as most frequently requested by hematologists and medical oncologists to determine lymphoma subtype and molecular profile (Figure 1) were, CD5 (87\%), CD10 (84\%), CD19 (83\%), BCL-2 (83\%) and the least frequently requested were CCND1 (44\%), CD15 (50\%), CD22 (65\%), and ALK (68\%). Three of the assays were more frequently requested by respondents from academic settings compared with those in nonacademic settings: CD10: 100\% vs $86 \%$ (chi-square; $P=.011$ ); CD30: $95 \%$ vs $80 \%$ (chi-square; $P=.028$ ); and MYC: $92 \%$ vs $70 \%$ (chi-square; $P=.004$ ).

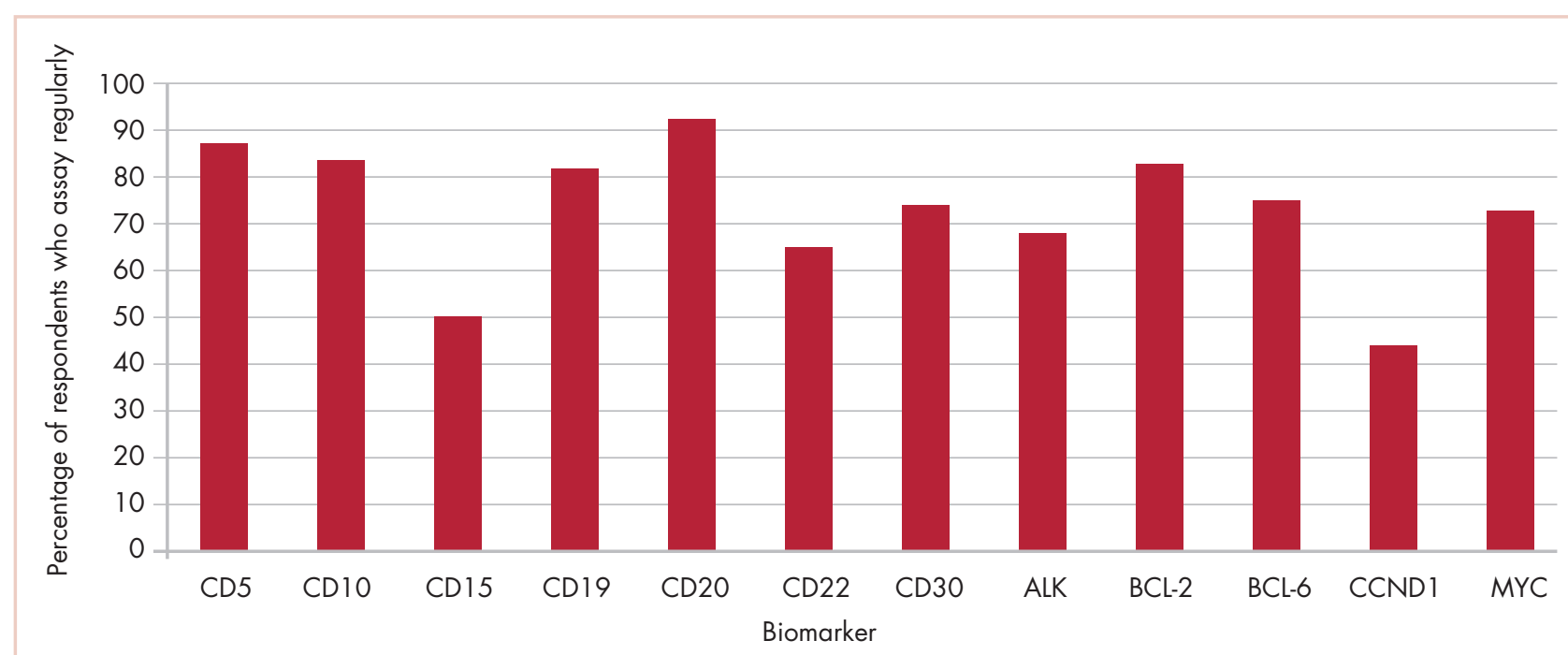

FIGURE 1 Routine practice regarding biomarker testing $(n=121)$. The assays reported as most frequently requested to determine lymphoma subtype and molecular profile were, CD5, CD10, and BCL-2. 
TABLE 4 Six questions hematologists and medical oncologists should ask themselves about their practice, based on the gaps identified in this study.

1. How familiar am I with recent guidelines updates and clinical trial data?

2. Am I subjecting my patients with chronic phase chronic myelogenous leukemia (CP-CML) to unnecessary bone marrow cytogenetic analysis in my attempt to monitor their response to tyrosine kinase inhibitor (TKI) therapy?

3. How confident am I of my interpretation of molecular and cytogenetic response data to inform treatment decisions in CP-CML?

4. How up-to-date is my knowledge of choice of therapy based on mutational analysis in the context of TKI resistance in CML?

5. To which level do I individualize therapy in patients with B-cell lymphomas or acute lymphoblastic leukemia, as opposed to using the therapy I am most comfortable with?

6. How familiar am I with currently promising investigational agents for hematologic malignancies?

Participants were asked questions about potential change in first-line therapy for a patient with diffuse large B-cell lymphoma (DLBCL) initially started on CHOP-R (cyclophosphamide, hydroxydaunorubicin, oncovin , prednisone - rituximab; Table 2, Questions 6-8). In Question 6, the patient scenario included an activated B-cell lymphoma genotype and $29 \%$ agreed with the expert recommendation to add bortezomib to CHOP-R. In Question 7, with both $M Y C$ and $B C L-2$ rearrangements included, $40 \%$ agreed with faculty recommendation to switch to EPOCH-R (etoposide, prednisone, vincristine, and doxorubicin hydrochloride - rituximab) and consider an autologous bone marrow transplant in first complete remission. In Question 8, a new scenario was explored for a patient with symptomatic mantle cell lymphoma. $\mathrm{CHOP}-\mathrm{R}$ was selected as initial therapy for this patient by $46 \%$ of the participants; whereas the expert recommendation was bendamustine and rituximab $(32 \%$ of respondents indicated that they would use this regimen). Qualitative interviews indicated that respondents were confused about indications and clinical data for therapies other than CHOP-R for patients with DLBCL or mantle cell lymphoma.

\section{Mechanisms of action for promising new agents}

Survey respondents were asked to match the generic names of 9 agents to their molecular target and only one, temsirolimus, was correctly matched to its molecular target by over $80 \%$ of the respondents (Figure 2). Only 2 other agents (brentuximab vedotin and aflibercept) had their targets correctly identified by over $60 \%$ of respondents. Five of the agents were correctly matched with their targets by less than a third of the participants. In addition, $65 \%$ of respondents got less than half of the answers correct, and only $7 \%$ correctly matched all 9 agents to their molecular target. Respondents with fewer than $10 \mathrm{CML}$, ALL, B-cell lymphoma cases a year had a mean of $28 \%$ correct answers, compared with a $45 \%$ mean for those with more than 10 cases per year.

\section{Discussion}

The challenges highlighted here are likely to reflect those faced by most hematologists and medical oncologists in the United States. The following discussion will demonstrate how knowledge and competency gaps could impact clinical efficiencies and patient outcomes and how practicing specialists, especially those who are community-based, can reflect on the existence of these gaps in their own practice. Table 4 provides questions that, based on the results of this study, could form the basis for clinical self-assessment, facilitating identification by hematologists and medical oncologists of their own clinical gaps, possibly leading them to seek educational strategies that could improve clinical efficiencies and patient outcomes.

When making first-line treatment choices for CML, hematologists and medical oncologists reported that they rely heavily on their previous experience with imatinib, suggesting that new data is not consistently being integrated into community practice. More specifically, new data indicates that early molecular response to TKI therapy is significantly associated with long-term survival outcomes, ${ }^{19,20}$ and that the second-generation TKIs (bosutinib, dasatinib, nilotinib) are superior to imatinib in relation to early efficacy responses. ${ }^{14,21-23} \mathrm{~A}$ better awareness and understanding of such data, through targeted education and expert guidance, may improve outcomes for patients with newly diagnosed chronic phase CML.

Many community specialists encounter a limited number of CML cases annually and therefore, assaying and interpreting response to first-line TKI therapy is challenging. Until recently, bone marrow cytogenetic analysis had been the gold standard for monitoring response to TKI therapy in chronic phase (CP-) CML. However, more experts and guideline recommendations have begun to rely on molecular responses by QPCR from peripheral blood every 3 months as important milestones for response and predicting long-term outcomes for their patients. ${ }^{14,24,25}$ This study indicates that bone marrow cytogenetic analysis is being used more frequently than recommended. The overuse of invasive bone marrow cytogenetic analysis could have multiple consequences to patient quality of life, patient adherence to their recommended monitoring schedule, and use of resources and cost to health care systems.

These findings suggest that most hematologists and medical oncologists are misaligned with expert-recommended practice on when to suggest a change in TKI therapy. NCCN14 and ELN17 Guidelines recommend con- 


\section{Original Report}

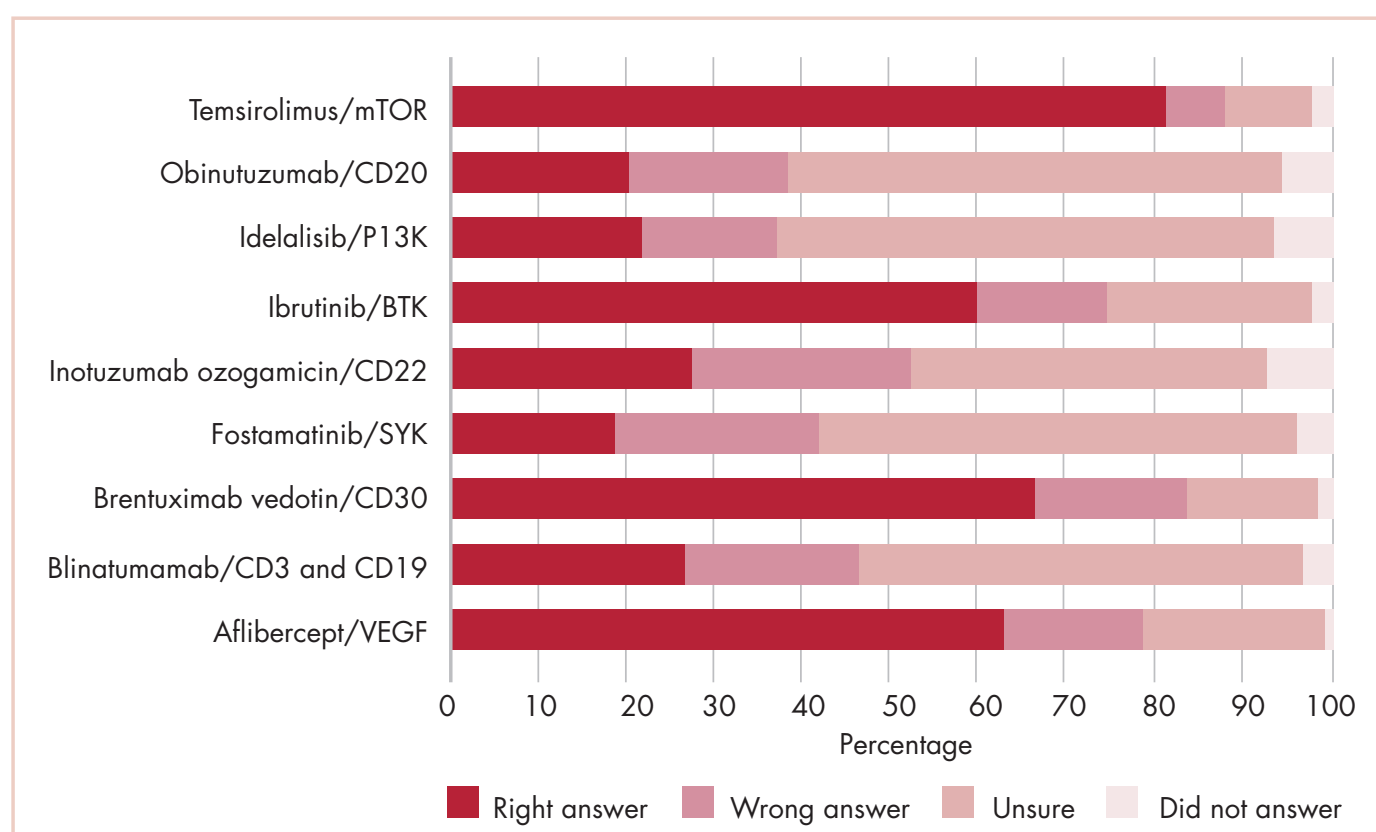

FIGURE 2 Answers to the 9 questions asking participants to match the therapeutic agent with its target $(\mathrm{n}=$ 121). Only 2 other agents (brentuximab vedotin and aflibercept) had their targets correctly identified by more than $60 \%$ of respondents.

ment options besides ponatinib may also overcome secondary TKI resistance.

The results from this national assessment indicate that CHOP-R combination chemotherapy is still the standard choice for newly diagnosed patients with aggressive B-cell lymphomas in many practices despite growing evidence for individualized approaches for different subpopulations of patients with biologically distinct variants. ${ }^{16,26,27}$ For example, translocations that target the oncogenes MYC and BCL2 have been consistently reported and there is consensus that MYC translocations with or without BCL2 (so-called double-hit mutations) confer a worse

sideration of a therapeutic change if $\mathrm{BCR}-\mathrm{ABL} / \mathrm{ABL}$ is > $10 \%$ on the International Scale at 3 or 6 months. Findings from the study suggest that this recommendation is not well integrated by hematologists and oncologists in the United States because almost half of the respondents would not consider a change in therapy for a patient with BCR-ABL/ABL of $15 \%$ on the International Scale and $17 / 20 \mathrm{Ph}$-positive metaphases at 3 months. The subgroup difference observed in relation to the hematologic case load of the participants exemplifies how rareness of the disease impedes adoption of best practices.

Guidelines recommend performing mutational analysis in the context of poor response to first-line therapy likely due to secondary TKI resistance and suggest that the choice of therapeutic strategy to overcome TKI resistance should be based in part on the results of a mutation analysis. ${ }^{14,17,24}$ Findings from this study indicate that a substantial proportion of hematologists and medical oncologists are challenged to interpret mutational analysis reports and to select second-line or salvage therapy. Many community specialists do not fully understand that the available TKIs have unique resistance profiles and that some mutations (other than T315I) may prompt selection of a particular TKI. However, many BCR-ABL mutations do not instill resistance to available TKIs. Therefore education is needed to reinforce that a stem cell transplant is likely unnecessary following failure of first-line therapy for patients with CP-CML and that for mutations other than T315I, treat- prognosis in patients with DLBCL who are treated with CHOP-R ${ }^{26,28}$ Phase 3 clinical trial data indicate that the combination of bendamustine and rituximab is likely to be better tolerated and more effective than CHOP-R for patients with mantle cell lymphoma and both CHOP-R and bendamustine and rituximab are recommended in the NCCN guidelines. ${ }^{16}$

Findings suggest that a key part of treatment decisionmaking related to patients with ALL and B-cell lymphomas is often empirical, largely relying on clinical experience with each treatment option. Although balancing risks and benefits is always part of the art of medicine, this study indicates that many community hematologists and medical oncologists are not individualizing therapy for patients with ALL and B-cell lymphomas, potentially explaining the variability in their patients' response.

A lack of knowledge of the unique mechanisms of action of emerging experimental agents for hematologic malignancies may help explain why, despite guidelines recommending clinical trials for patients with relapsed or refractory ALL and B-cell lymphomas, enrollment in clinical trials for these tumors remain low. Furthermore, this trend may be even more pronounced in these diseases as many patients are either young adults or elderly.29 Moreover, a lack of familiarity with generic names, mechanisms of action, and biologic rationale for use may lead to missed opportunities to enroll eligible patients in clinical trials. Of more importance, hematologists and medical oncologists 
without a complete understanding of these agents will lack competence to effectively apply emerging clinical trial data and agents with new indications into their clinical practice.

\section{Limitations}

Self-selection bias was a possibility, as participation in the study was voluntary, but the use of purposive sampling improves the probability of having a sample that is representative of the targeted population. ${ }^{11} \mathrm{~A}$ subset of participants $(n=71)$ from which demographic information was incomplete was removed from the analysis to avoid inclusion of potentially noneligible participants. All results are based on self-report by the participants and the relation to actual practice is assumed. Optimally, these results would be compared to chart-level data. In the future it will be interesting to see if these data are reflected in treatment databases.

\section{Conclusions}

This study has identified important areas of practice where performance gaps among US hematologists and medical oncologists may be hindering delivery of optimal care to patients with CML, ALL, or B-cell lymphomas. Two common points across the findings presented here raise questions that go beyond the precise clinical points tested. First, it illustrates the increasing complexity of treatment decisions as more treatment options become available. Second it raises the question of how physicians can stay current on specific low prevalence diseases that represent a small percentage of their case load.

Our findings should be considered in the design of continuing professional development and educational programs. In addition, within the context of the US PPACA, which increases pressure for greater efficiency in the delivery of healthcare services, our findings could stimulate selfreflection among community hematologists and medical oncologists on knowledge or competency gaps that may exist in their own practice, and incite them to deploy local educational and performance improvement strategies.

\section{Acknowledgments}

This study was conducted by Annenberg Center for Health Sciences at Eisenhower, Axdev Group Inc, and Clinical Care Options LLC. The sponsor had no role in study design; in the collection, analysis and interpretation of data; in the writing of the report; and in the decision to submit the article for publication. The authors acknowledge the support that was provided by Sean M Hayes, the general manager, and Sophie Péloquin, the performance improvement researcher, at Axdev Group. The authors also thank the hematologists and medical oncologists who participated in the study.

\section{References}

1. John S, Niederhuber JE. Keeping pace. Oncologist. 2008;13:4-5.

2. Cultrera JL, Dalia SM. Diffuse large B-cell lymphoma: current strategies and future directions. Cancer Control. 2012;19:204-213.

3. Jain P, Kantarjian H, Cortes J. Chronic myeloid leukemia: overview of new agents and comparative analysis. Curr Treat Options Oncol.
2013;14:127-143.

4. Klepin HD, Rizzieri D, Palumbo A, Magarotto V, Eichhorst B. Individualizing treatment decisions for older adults with hematologic malignancies. Am Soc Clin Oncol Educ Book. 2013;33:208-219.

5. Zhao $\mathrm{Y}$, Huang $\mathrm{H}$, Wei $\mathrm{G}$. Novel agents and biomarkers for acute lymphoid leukemia. J Hematol Oncol. 2013;6:40.

6. Association of Community Cancer Centers. Final report: treating small-population cancers in the community setting. http://www.accccancer.org/education/CML-FinalReport.asp. Released January 30 2013. Accessed October 17, 2013

7. Government Printing Office, US Government. The Patient Protec tion and Affordable Care Act (PPACA). Public Law 1482010

8. Creswell JW, Klasse AC, Plano VLC, Smith KC. Best practices for mixed methods research in the health sciences. National Institutes of Health. http://obssr.od.nih.gov/mixed_methods_research. Released August 2011. Accessed October 17, 2013.

9. Maudsley G. Mixing it but not mixed-up: mixed methods research in medical education. Medical teacher. 2011;33:e92-104

10. US Department of Health and Human Services. Ethical Principles and Guidelines for the Protection of Human Subjects of Research. http://www.hhs.gov/ohrp/humansubjects/guidance/belmont.html Released April 18, 1979. Accessed October 17, 2013

11. Given LM (ed). The Sage Encyclopedia of Qualitative Research Methods. Sage Publications: Thousand Oaks, CA. 2008.

12. Boyatzis R. Thematic analysis and code development: transform ing qualitative information. Sage publications: Thousand Oaks, CA. 1998.

13. Hsieh HF, Shannon SE. Three approaches to qualitative content analysis. Qual Health Res. 2005;15:1277-1288

14. National Comprehensive Cancer Network. Clinical practice guidelines in oncology: chronic myelogenous leukemia. 2013. http://www. nccn.org. Accessed October 17, 2013.

15. National Comprehensive Cancer Network. Clinical practice guidelines in oncology: acute lymphoblastic leukemia. 2013. http://www. nccn.org. Accessed October 17, 2013.

16. National Comprehensive Cancer Network. Clinical practice guide lines in oncology: Non-Hodgkin's lymphomas. 2013. http://www. nccn.org. Accessed October 17, 2013.

17. The European LeukemiaNet (ELN). Management of chronic myeloid leukemia (CML): recommendations from the European LeukemiaNet (ELN). 2013. http://www.leukemia-net.org/content/leukemias/cml/recommendations/index_eng.html. Accessed October 17,2013

18. Moore D. Needs assessment in the new healthcare environment: combining discrepancy analysis and outcomes to create more effective CME. J Contin Educ Health Prof. 1998;18:133-141.

19. Brümmendorf TH, Kantarjian HM, Gambacorti-Passerini C et al. Assessment of early molecular response as a predictor of long-term clinical outcomes in the phase 3 BELA study. Paper presented at: American Society of Hematology Annual Meeting; December 8-11, 2012; Atlanta, Georgia.

20. Marin D, Ibrahim AR, Lucas C, et al. Assessment of BCR-ABL1 transcript levels at 3 months is the only requirement for predicting outcome for patients with chronic myeloid leukemia treated with tyrosine kinase inhibitors. J Clin Oncol. 2012;30:232-238.

21. Hochhaus A, Hughes TP, Saglio G. Outcome of patients with chronic myeloid leukemia in chronic phase (CML-CP) based on early molecular response and factors associated with early response: 4-year follow-up data from ENESTnd (Evaluating Nilotinib Efficacy and Safety in Clinical Trials Newly Diagnosed Patients). Paper presented at: American Society of Hematology Annual Meeting; December 8-11, 2012; Atlanta, Georgia.

22. Kantarjian H, Finn I, Goldberg S. Nilotinib versus imatinib in patients with newly diagnosed chronic myeloid leukemia in chronic phase: ENESTnd 3-year follow-up. Paper presented at: American Society of Clinical Oncology Annual Meeting; June 1-5, 2012; Chicago, Illinois.

23. Cortes JE, Kim D-W, Kantarjian HM, et al. Bosutinib Versus Imatinib in Newly Diagnosed Chronic-Phase Chronic Myeloid Leukemia: Results From the BELA Trial. J Clin Oncol. 2012;30:3486 3492. 


\section{Original Report}

24. Cortes J, Goldman JM, Hughes T. Current issues in chronic myeloid leukemia: monitoring, resistance, and functional cure. JNCCN 2012;10 Suppl 3:S1-S13.

25. Jabbour E, Kantarjian H. Chronic myeloid leukemia: 2012 update on diagnosis, monitoring, and management. Am J Hematol. 2012;87:1037-1045.

26. Barrans S, Crouch S, Smith A, et al. Rearrangement of MYC Is Associated With Poor Prognosis in Patients With Diffuse Large BCell Lymphoma Treated in the Era of Rituximab. J Clin Oncol. 2010;28:3360-3365.
27. Jaffe ES, Pittaluga S. Aggressive B-Cell Lymphomas: A Review of New and Old Entities in the WHO Classification. Hematology Am Soc Hematol Educ Program. 2011;1:506-514.

28. Savage KJ, Johnson NA, Ben-Neriah S, et al. MYC gene rearrangements are associated with a poor prognosis in diffuse large B-cell lymphoma patients treated with R-CHOP chemotherapy. Blood. 2009;114:3533-3537.

29. Padberg R. Cancer Clinical Trial Accrual: We have a problem. J Canc Educ. 2011;26:403-404. 